\title{
Discrimination and reversal in capuchin monkeys as a function of irrelevant cue salience
}

\author{
JOHN L. SCANLON and JAMES E. KING \\ University of Arizona, Tucson, Arizona 85721
}

\begin{abstract}
Eight capuchin monkeys were trained on a simultaneous shape discrimination and a subsequent reversal with size, brightness, and position cues irrelevant. One group (HS) was trained with highly salient size and brightness cues, and the other group (LS) was trained with less salient size and brightness cues. The two groups did not differ significantly in learning either the original discrimination or its reversal. However, during reversal, Group HS responded more to its preferred size than did Group LS. This result indicates that the emergence of previously absent preferences along an irrelevant dimension is a function of the salience of the dimension. Responding to irrelevant dimensions was also found to increase from acquisition to reversal for the HS group, but not for the LS group. This increase is not consistent with Mackintosh's recent theory of discrimination learning.
\end{abstract}

A variety of animal learning experiments have amply demonstrated that responses to irrelevant dimensions do not necessarily decrease monotonically as learning proceeds. For example, Turner (1968) reported that position habits increased during acquisition of brightness discrimination by rats in a Lashley jumping stand, and Shepp and Schrier (1969) obtained comparable results during learning set acquisition by macaque monkeys. Increased position responding was also previously observed by the authors (Scanlon \& King, 1976) in sameness-difference learning by capuchin monkeys. In addition, previously suppressed position habits and other systematic modes of responding correlated with irrelevant dimensions are potentially prone to elicitation during reversal of a previously learned discrimination, a phenomenon that Harlow (1959) has referred to as "regression." An example of regression was the appearance during reversal of stimulus preference and differential cue error factors on a series of multiple discrimination reversal problems (Harlow, 1950).

Increased responding to irrelevant dimensions during acquisition and regression during reversal are inconsistent with a recent theory of discrimination learning proposed by Mackintosh (1975). According to this theory, a dimension-specific learning rate parameter, $\alpha$, changes for each stimulus dimension according to that dimension's correlation with reinforcement. Increases in $\alpha_{A}$ occur only when Dimension A is correlated with reinforcement and, as in overshadowing, is more salient than another stimulus dimension also correlated with reinforcement, or, as in blocking, has been previously established as the signal for the reinforcer. Conversely, $\alpha_{B}$ decreases only when Dimension B is not correlated

This research was partially supported by Training Grant MH-11286 from the U.S. Public Health Service. Requests for reprints should be sent to James E. King, Department of Psychology, University of Arizona, Tucson, Arizona 85721. with reinforcement or when Dimension B predicts no change in reinforcement that has been already or more saliently signaled by Dimension A. A change for $\alpha$ for one dimension does not, however, cause a change in $\alpha$ for other dimensions.

Mackintosh's (1975) theory is largely based on results of blocking and overshadowing procedures, both of which entail the use of redundantly relevant stimulus dimensions. An alternative experimental procedure is to manipulate the salience of the irrelevant dimensions, while holding the salience of a single relevant dimension constant.

According to Mackintosh's (1975) new theory, since the only signal correlated with reinforcement is held constant, the learning rate should be the same, regardless of the saliences of the irrelevant dimensions. However, since the irrelevant dimensions remain uncorrelated with reinforcement, preferences along these dimensions should not increase because of increases in their salience.

These two predictions are not consequences of selective attention theories of discrimination learning (e.g., Lovejoy, 1968; Sutherland \& Mackintosh, 1971; Trabasso \& Bower, 1968; Zeaman \& House, 1963), all of which incorporate what Thomas (1970) referred to as the "inverse hypothesis." According to the inverse hypothesis, attention to different dimensions of a stimulus array are not independent but are inversely related; an increase in attention to one dimension necessarily requires a decrease in attention to one or more other dimensions.

The experiment described below was designed to permit manipulation of the salience of two irrelevant dimensions (size and brightness), while holding constant the salience of the relevant dimension (shape). Irrelevant dimensions were made more salient for one group of capuchin monkeys by selecting cue values further apart on the dimensional continua. 
In addition to initial training on the shape discrimination, all subjects were trained on its reversal. Because of the regression phenomenon previously noted, we expected that attention to the irrelevant dimensions of size and brightness would increase following the reversal, especially for the group trained on irrelevant dimensions that were highly salient. A resurgence of responding to the irrelevant dimension would be consistent with theories that assume some form of the inverse hypothesis, since a reversal would probably cause an immediate, temporary drop in attention to the relevant dimension. Mackintosh's (1975) theory, however, would be unable to explain an increase in attention to dimensions consistently uncorrelated with reinforcement.

\section{METHOD}

\section{Subjects}

Subjects were four male and four female capuchin monkeys (Cebus apella) with previous experience on a variety of object discriminations. Two males and two females were randomly assigned to each of the two training groups.

\begin{abstract}
Apparatus
All testing was conducted with a manually operated Wisconsin General Test Apparatus (WGTA). The stimulus tray on which the stimulus objects were placed measured $15 \times 35.5 \mathrm{~cm}$ and contained two food wells located $18 \mathrm{~cm}$ apart from center to center. Stimulus objects used during adaptation were four junk objects attached to flat $4.2 \times 4.2 \mathrm{~cm}$ plywood bases. Objects used during acquisition and reversal were wood squares and rectangles mounted at right angles to the front of $4.2 \times 4.2 \mathrm{~cm}$ plywood bases. The squares and rectangles were three different sizes: small (area approximately $3.65 \mathrm{~cm}^{2}$, with squares measuring $1.8 \times 1.8 \mathrm{~cm}$ and rectangles measuring $2.5 \times 1.5 \mathrm{~cm}$ ), medium (area approximately $6.25 \mathrm{~cm}^{2}$, with squares measuring $2.5 \times 2.5 \mathrm{~cm}$ and rectangles measuring $3.3 \times 1.9 \mathrm{~cm}$ ), and large (area approximately $19.4 \mathrm{~cm}^{2}$, with squares measuring $4.4 \times 4.4 \mathrm{~cm}$ and rectangles measuring $5.9 \times 3.3 \mathrm{~cm}$ ). Except for the faces of the squares and rectangles, which were white, light gray, or black, the objects and bases were painted a uniform black.
\end{abstract}

\section{Testing Procedure}

At the start of each trial, the forward opaque screen of the WGTA was raised and the stimulus presentation tray was slowly moved forward toward the subject. Displacement of correct object revealed one-half of a raisin in the food well. Correction was not allowed. The screen was lowered after the subject's response, and the tray was then withdrawn. The position of the positive stimulus was varied semirandomly, with the restriction that all eight possible 3-trial position sequences appeared exactly three times on successive 72-trial blocks.

\section{Experimental Design}

During adaptation, the monkeys were trained on two objectdiscrimination problems with the previously described junk stimuli. Each of the two problems was presented for 24 trials/ day until each monkey achieved a criterion of 9 out of 10 correct responses.

\section{Acquisition and Reversal}

During acquisition, half of the animals in each group were rewarded for responses to squares and half for responses to rectangles. During reversal, the opposite cue for each subject was rewarded. All subjects were presented four basic types of stimulus displays, each of which was given with the correct object on the left and on the right equally often. Size and brightness cues were uncorrelated with reinforcement; that is, the smaller object was rewarded as often as the larger object, and the brighter object was rewarded as often as the darker object.

The LS group, trained with size and brightness dimensions of low salience, received the following four types of stimulus displays: (1) a small white square and a medium gray rectangle, (2) a medium white square and a small gray rectangle, (3) a medium gray square and a small white rectangle, and (4) a small gray square and a medium white rectangle. The HS group, trained with highly salient size and brightness cues, were presented the four types of stimulus displays as described above, but with large rather than medium size cues and with black rather than gray brightness cues.

Thirty-six trials were presented daily until the criterion of 35 correct responses on 1 day's testing was achieved for the acquisition phase. This procedure was repeated for the reversal phase, which began on the day following completion of the original shape discrimination.

\section{RESULTS}

Analysis of variance revealed no significant group, phase, or Group by Phase interaction effects for either the number of days or number of errors to criterion.

The strength of responding to each dimension (shape, size, brightness, and position) was assessed by measuring each subject's preference for its preferred cue value within that dimension. This simple bias is given by the formula $2(P)-1$, where $P$ is the probability of choosing the preferred cue. If the two cues were equally preferred, no bias would be present, since $2(.5)-1=0$. Similarly, if one cue was preferred on all trials, the bias would be 1.0 , since $2(1.0)-1=1.0$. In addition, each observed bias was subjected to a z-transformation to determine if that value differed significantly $(p<.05)$ from zero. If it did not, it was assumed to be caused by chance alone and was recorded as zero bias. The response bias for each dimension was calculated for each 144-trial block for each subject. Figure 1 shows the mean strength of the response bias of each group for each dimension during the acquisition and reversal phases. Separate analyses of variance were applied to the response-bias values for each of the four stimulus dimensions, as well as for size and brightness combined.

Overall analysis of responding to the relevant shape dimension revealed no significant group, phase, or interaction effects. However, data from the last block of acquisition and the first block of reversal showed a significant drop in responding to the shape dimension from one phase to the next $[F(1,6)=17.25, p<.01]$. Analysis of position responding indicated no significant group, phase, or group by phase interaction effects.

For the irrelevant size dimension, group and phase effects were not significant, but a significant interaction between these two variables occurred $[F(1,6)=6.411$, $\mathrm{p}<.05]$. Further analysis of this interaction showed significantly more size responding by the HS group 


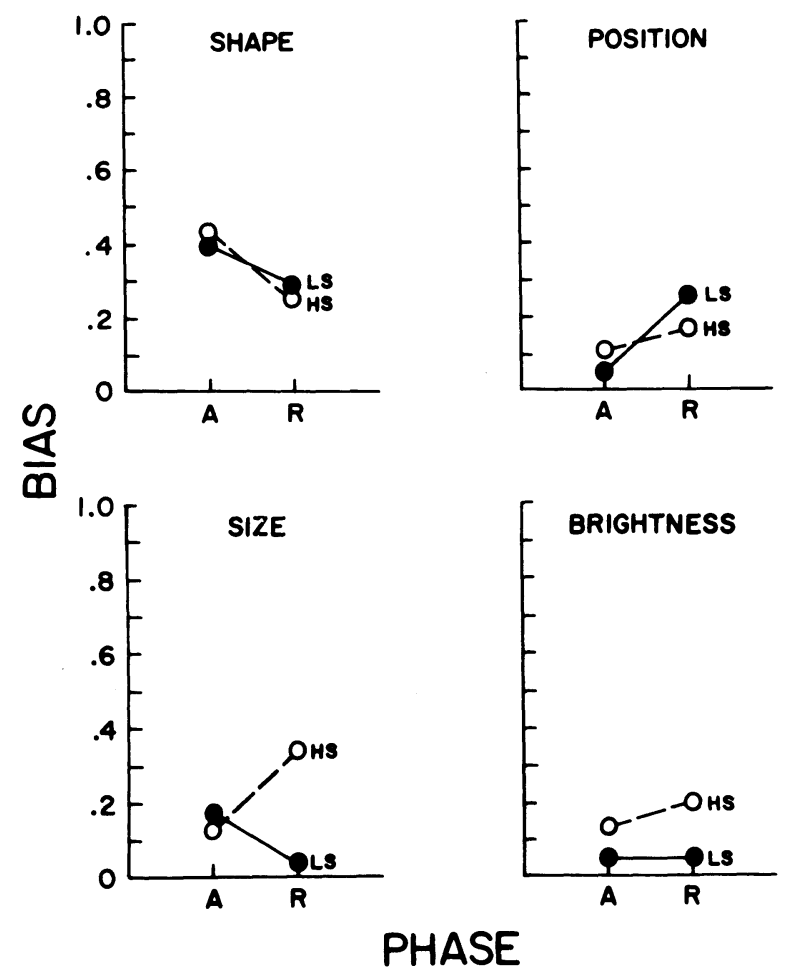

Figure 1. Strength of biases in high- and low-salience conditions during acquisition $(A)$ and reversal $(R)$.

than by the LS group during reversal $[\mathrm{t}(6)=2.877$, $\mathrm{p}<.025$ ], but not during acquisition. Results for the analysis of brightness responding indicated more brightness responses by the HS group than by the LS group $[F(1,6)=8.403, p<.05]$, but no significant phase or interaction effects.

When size and brightness responses were combined, the HS group again demonstrated more responding to these irrelevant cues than did the LS group $[F(1,6)=$ $10.930, p<.025]$. Further analysis revealed that the LS group responded to these cues less than did the HS group during reversal $[t(6)=5.845, p<.01]$, but not during acquisition. These results are the same as those found for size responding alone. However, unlike the analysis of size or brightness alone, the combination of the two showed that the HS group responded to these cues more during reversal than during acquisition $[t(3)=2.795, p<.05]$. This difference was not found for the LS group.

\section{DISCUSSION}

The results of this experiment failed to reveal any significant retardation of either the acquisition of the shape discrimination or its reversal as a result of increased salience of the irrelevant size and brightness dimensions. This result is consistent with
Mackintosh's (1975) new theory, since the salience of the relevant shape dimension was the same for both groups of monkeys. However, analysis of responses made to the preferred and irrelevant size and brightness cues revealed interesting differences between high- and low-salience conditions. The group for which these irrelevant dimensions were most salient manifested enhanced responding to size and brightness, especially during the reversal phase. This group also increased its responding to these irrelevant cues from acquisition to reversal, which is consistent with Harlow's (1950) demonstration of the resurgence of previously suppressed error factors during reversal performance.

This last finding, in which responding to dimensions consistently uncorrelated with reinforcement increased, cannot be explained by Mackintosh's (1975) theory. According to this theory, such an increase should only be expected for dimensions correlated with reinforcement (i.e., relevant dimensions). This finding, however, is consistent with theories that assume an inverse hypothesis. Since responding to the relevant shape dimension underwent a significant drop during the transition from acquisition to reversal, the observed increase in responding to one or more other dimensions would be expected according to the inverse hypothesis.

During reversal, the monkeys' enhanced responsiveness to the high-salience size and brightness cues demonstrated that the regression phenomenon is a function of irrelevant cue salience. Furthermore, this result showed that regression is a general effect, not limited to elicitation by cues, such as position or preferences for particular complex objects, which are highly salient at the start of acquisition.

\section{REFERENCES}

Harlow, H. F. Performance of catarrhine monkeys on a series of discrimination reversal problems. Journal of Comparative and Physiological Psychology, 1950, 43, 231-239.

HarLOW, H. F. Learning set and error factor theory. In S. Koch (Ed.), Psychology: A study of a science (Vol. 2). New York: McGraw-Hill, 1959.

Lovejoy, E. Attention in discrimination learning. San Francisco: Holden-Day, 1968.

Mackintosh, N. J. A theory of attention: Variations in the associability of stimuli with reinforcement. Psychological Review, 1975, 82, 276-298.

Scanlon, J. L., \& KING, J. E. Learning and transposition of an extended sameness-difference concept by slow and fast learning capuchin monkeys. Animal Learning \& Behavior, 1976, 4, 308-312.

She PP, B. E., \& Schrier, A. M. Consecutive intradimensional and extradimensional shifts in monkeys. Journal of Comparative and Physiological Psychology, 1969, 67, 199-203.

Suthe Rland, N. S., \& Mackintosh, N. J. Mechanisms of animal discrimination learning. New York: Academic Press, 1971.

Tномаs, D. R. Stimulus selection, attention, and related matters. In J. H. Reynierse (Ed.), Current issues in animal learning. Lincoln: University of Nebraska Press, 1970.

Trabasso, T., \& Bower, G. H. Attention in learning: Theory and research. New York: Wiley, 1968.

TURner, C. Models of discrimination learning. Unpublished doctoral thesis, Oxford University, 1968.

Zeaman, D., \& House, B. The role of attention in retardate discrimination learning. In N. R. Ellis (Ed.), The handbook of mental deficiency. New York: McGraw-Hill, 1963.

(Received for publication May 8, 1980.) 